PROCEEDINGS OF THE

AMERICAN MATHEMATICAL SOCIETY

Volume 131, Number 12, Pages 3883-3892

S 0002-9939(03)07074-6

Article electronically published on April 24, 2003

\title{
ISOMORPHISMS OF SUBALGEBRAS OF NEST ALGEBRAS
}

\author{
FANGYAN LU
}

(Communicated by David R. Larson)

\begin{abstract}
Let $\mathcal{T}$ be a subalgebra of a nest algebra $\mathcal{T}(\mathcal{N})$. If $\mathcal{T}$ contains all rank one operators in $\mathcal{T}(\mathcal{N})$, then $\mathcal{T}$ is said to be large; if the set of rank one operators in $\mathcal{T}$ coincides with that in the Jacobson radical of $\mathcal{T}(\mathcal{N}), \mathcal{T}$ is said to be radical-type. In this paper, algebraic isomorphisms of large subalgebras and of radical-type subalgebras are characterized. Let $\mathcal{N}_{i}$ be a nest of subspaces of a Hilbert space $\mathcal{H}_{i}$ and $\mathcal{T}_{i}$ be a subalgebra of the nest algebra $\mathcal{T}\left(\mathcal{N}_{i}\right)$ associated to $\mathcal{N}_{i}(i=1,2)$. Let $\phi$ be an algebraic isomorphism from $\mathcal{T}_{1}$ onto $\mathcal{T}_{2}$. It is proved that $\phi$ is spatial if one of the following occurs: (1) $\mathcal{T}_{i}(i=1,2)$ is large and contains a masa; (2) $\mathcal{T}_{i}(i=1,2)$ is large and closed; (3) $\mathcal{T}_{i}(i=1,2)$ is a closed radical-type subalgebra and $\mathcal{N}_{i}(i=1,2)$ is quasi-continuous (i.e. the trivial elements of $\mathcal{N}_{i}$ are limit points); (4) $\mathcal{T}_{i}(i=1,2)$ is large and one of $\mathcal{N}_{1}$ and $\mathcal{N}_{2}$ is not quasi-continuous.
\end{abstract}

\section{INTRODUCTION AND PRELIMINARIES}

A nest $\mathcal{N}$ is a totally ordered set of closed subspaces of a Hilbert space $\mathcal{H}$ containing (0) and $\mathcal{H}$ which is closed under intersection and closed span. By $B(\mathcal{H})$, we denote the set of all linearly bounded operators on $\mathcal{H}$, and if $\mathcal{H}_{1}$ and $\mathcal{H}_{2}$ are Hilbert spaces, then $B\left(\mathcal{H}_{1}, \mathcal{H}_{2}\right)$ denotes the set of all linearly bounded operators from $\mathcal{H}_{1}$ to $\mathcal{H}_{2}$. The nest algebra denoted by $\mathcal{T}(\mathcal{N})$ associated to $\mathcal{N}$ is the set of operators in $B(\mathcal{H})$ which leave every element in $\mathcal{N}$ invariant. For $E \in \mathcal{N}$, we use $E$ to denote both a subspace and the orthogonal projection to it. So $E^{\perp}$ denotes the orthogonal complement $\mathcal{H} \ominus E$ and the difference $I-E$. Given a nest $\mathcal{N}$ and $E \in \mathcal{N}$, define $E_{-}=\bigvee\{N: N<E, N \in \mathcal{N}\}$ and $E_{+}=\bigwedge\{N: N>E, N \in \mathcal{N}\}$ $\mathcal{N}$ is continuous if $E_{-}=E$ for each $E \in \mathcal{N} ; \mathcal{N}$ is quasi-continuous if $(0)_{+}=(0)$ and $\mathcal{H}_{-}=\mathcal{H} ; \mathcal{N}$ is maximal if $\operatorname{dim}\left(E \ominus E_{-}\right) \leq 1$ for every $E \in \mathcal{N} ; \mathcal{N}$ is sub-maximal if $\operatorname{dim}\left((0)_{+}\right) \leq 1$ and $\operatorname{dim}\left(\mathcal{H} \ominus \mathcal{H}_{-}\right) \leq 1$.

Let $x$ and $y$ be non-zero vectors in $\mathcal{H}$. Then rank one operator $x \otimes y$ is defined by $(x \otimes y) z=(z, y) x$ for any $z \in \mathcal{H}$. Let $\mathcal{T}(\mathcal{N})$ be a nest algebra and $\mathcal{R}_{\mathcal{N}}$ be the Jacobson radical of $\mathcal{T}(\mathcal{N})$. It is well known that $x \otimes y$ belongs to $\mathcal{T}(\mathcal{N})$ if and only if there is $E$ in $\mathcal{N}$ such that $x \in E$ and $y \in E_{-}^{\perp}$, and to $\mathcal{R}_{\mathcal{N}}$ if and only if there is $E$ in $\mathcal{N}$ such that $x \in E$ and $y \in E^{\perp}$. Thus $\mathcal{R}_{\mathcal{N}}$ contains all rank one operators in $\mathcal{T}(\mathcal{N})$ only when $\mathcal{N}$ is continuous.

Received by the editors September 24, 2001 and, in revised form, August 8, 2002.

2000 Mathematics Subject Classification. Primary 47L75, 47L35.

Key words and phrases. Algebraic isomorphisms, nest algebras, large subalgebras, radical-type algebras, rank one operators, spatially implemented. 
Definition 1.1. Let $\mathcal{T}$ be a subalgebra of $\mathcal{T}(\mathcal{N})$. If $\mathcal{T}$ contains all rank one operators in $\mathcal{T}(\mathcal{N})$, then we say that $\mathcal{T}$ is large. If the set of rank one operators in $\mathcal{T}$ coincides with that in the Jacobson radical of $\mathcal{T}(\mathcal{N})$, we say that $\mathcal{T}$ is radicaltype.

In the fundamental work [6] on the theory of nest algebras, R. Ringrose established the Isomorphism Theorem of nest algebras which states that every algebraic isomorphism between two nest algebras is necessarily spatial. In the same paper, he also proved that

Theorem 1.2 ([, Theorem 4.1]). Let $\mathcal{N}_{i}$ be a nest of subspaces of a Hilbert space $\mathcal{H}_{i}$ and $\mathcal{T}_{i}$ be a subalgebra of nest algebra $\mathcal{T}\left(\mathcal{N}_{i}\right)$ associated to $\mathcal{N}_{i}(i=1,2)$. Let $\phi$ be an algebraic isomorphism from $\mathcal{T}_{1}$ onto $\mathcal{T}_{2}$. Suppose that (1) $\mathcal{T}_{i}$ is large; (2) there is a maximal abelian ${ }^{*}$-subalgebra (masa) $\mathcal{A}_{i}$ of $B\left(\mathcal{H}_{i}\right)$ in $\mathcal{T}_{i} ;(3) \phi\left(\mathcal{A}_{1}\right)=\mathcal{A}_{2}$. Then $\phi$ is spatially implemented, i.e. there is an invertible operator $S \in B\left(\mathcal{H}_{1}, \mathcal{H}_{2}\right)$ such that $\phi(T)=S T S^{-1}$ for every $T \in \mathcal{T}_{1}$.

As we observed, the Isomorphism Theorem of nest algebras is a corollary of Theorem 1.2, since in this case the isomorphism is the composition of two isomorphisms, one of which is spatial and the other satisfies hypotheses (1)-(3) in Theorem 1.2 [1. Theorem 17.5]. This suggests that condition (3) in Theorem 1.2 may be removed for appropriate subalgebras. In fact, in [3] we proved

Theorem 1.3 ([3, Theorem 3.4]). Let $\mathcal{N}_{i}$ be a nest of subspaces of a Hilbert space $\mathcal{H}_{i}$ and $\mathcal{T}_{i}$ be a subalgebra of nest algebra $\mathcal{T}\left(\mathcal{N}_{i}\right)$ associated to $\mathcal{N}_{i}(i=1,2)$. Let $\phi$ be an algebraic isomorphism from $\mathcal{T}_{1}$ onto $\mathcal{T}_{2}$. Suppose that $(1) \mathcal{H}_{i} \ominus\left(\mathcal{H}_{i}\right)_{-}$and $\left(0_{i}\right)_{+}$both have dimension $\leq 1 ;(2)$ there is a maximal abelian ${ }_{-}^{*}$-subalgebra (masa) $\mathcal{A}_{i}$ of $B\left(\mathcal{H}_{i}\right)$ in $\mathcal{T}_{i}$; (3) the invariant subspace lattice of $\mathcal{T}_{i}$ is $\mathcal{N}_{i}$; (4) $\mathcal{T}_{i}$ contains every rank one operator $x \otimes y$ with $x \in E$ and $y \in E^{\perp}$ for some element $E$ in $\mathcal{N}_{i}$. Then $\phi$ is spatially implemented.

In the present paper, we continue to investigate algebraic isomorphisms of subalgebras of nest algebras. We pay our attention to algebraic isomorphisms of large subalgebras and of radical-type subalgebras. Unlike Theorem 1.2 and Theorem 1.3 , we do not require subalgebras to contain masas. If subalgebras contain masas, unlike Theorem 1.2 and like Theorem 1.3, we do not require the algebraic isomorphism to satisfy condition (3) in Theorem 1.2. Let $\mathcal{N}_{i}$ be a nest of subspaces of a Hilbert space $\mathcal{H}_{i}$ and $\mathcal{T}_{i}$ be a subalgebra of nest algebra $\mathcal{T}\left(\mathcal{N}_{i}\right)$ associated to $\mathcal{N}_{i}$ $(i=1,2)$. Let $\phi$ be an algebraic isomorphism from $\mathcal{T}_{1}$ onto $\mathcal{T}_{2}$. We will prove that $\phi$ is spatial if one of the following occurs: $(1) \mathcal{T}_{i}(i=1,2)$ is large and contains a masa; $(2) \mathcal{T}_{i}(i=1,2)$ is large and closed; $(3) \mathcal{T}_{i}(i=1,2)$ is large and one of $\mathcal{N}_{1}$ and $\mathcal{N}_{2}$ is not quasi-continuous; (4) $\mathcal{T}_{i}(i=1,2)$ is a closed radical-type subalgebra and $\mathcal{N}_{i}(i=1,2)$ is quasi-continuous. In particular, every algebraic isomorphism of compact operator ideals of nest algebras is spatial and so is every isomorphism of the Jacobson radicals of nest algebras associated to quasi-continuous nests.

In order to deal with isomorphisms of large subalgebras and of radical-type subalgebras simultaneously, we introduce $\theta$-subalgebras which are modeled on large subalgebras and radical-type subalgebras.

Definition 1.4. We say that a subalgebra $\mathcal{T}$ of $\mathcal{T}(\mathcal{N})$ is a $\theta$-subalgebra if

(1) $\theta$ is a homomorphism from the nest $\mathcal{N}$ to itself such that $\theta(E)=E$ or $E_{-}$ for every $E \in \mathcal{N}$. 
(2) A rank one operator $x \otimes y$ belongs to $\mathcal{T}$ if and only if there is $E \in \mathcal{N}$ such that $x \in E$ and $y \in \theta(E)^{\perp}$.

Thus a large subalgebra is a $\theta$-subalgebra where $\theta: E \rightarrow E_{-}$and the Jacobson radical is also a $\theta$-subalgebra where $\theta$ is the identity on $\mathcal{N}$. We would like to emphasize the following facts. For a $\theta$-subalgebra $\mathcal{T}$ of $\mathcal{T}(\mathcal{N}):(1)$ if $x \otimes y$ belongs to $\mathcal{T}$ and $E$ is the smallest element containing $x$ in $\mathcal{N}$, then $y \in \theta(E)^{\perp} ;(2)$ if $x \in E$ and $y \in E^{\perp}$, then $x \otimes y \in \mathcal{T}$.

In subsequent sections, isomorphisms always refer to algebraic isomorphisms.

\section{ISOMORPHISMS OF $\theta$-SUBALGEBRAS}

In this section, $\mathcal{N}_{i}$ is always a nest of subspaces of a Hilbert space $\mathcal{H}_{i}$ and $\mathcal{T}_{i}$ is a $\theta_{i}$-subalgebra of the nest algebra $\mathcal{T}\left(\mathcal{N}_{i}\right)(i=1,2) . \phi$ is an isomorphism from $\mathcal{T}_{1}$ onto $\mathcal{T}_{2}$. We use $\mathcal{N}_{i}^{\theta_{i}}$ to denote the set $\left\{E \in \mathcal{N}_{i}: E>(0)\right.$ and $\left.\theta_{i}(E)^{\perp} \neq(0)\right\}$.

Lemma 2.1. Suppose that $\bigvee\left\{\theta_{1}(E)^{\perp}: E \in \mathcal{N}_{1}^{\theta_{1}}\right\}=\mathcal{H}_{1}$ and $\phi$ preserves rank one operators. Let $E$ be in $\mathcal{N}_{1}^{\theta_{1}}$. If $y_{0}$ is a non-zero vector in $\theta_{1}(E)^{\perp}$, then there is $v_{0}$ with the property that for each $x \in E$ there exists a vector $u$ such that $\phi\left(x \otimes y_{0}\right)=$ $u \otimes v_{0}$.

Proof. Let $x$ be any non-zero vector in $E$. Then $x \otimes y_{0}$ belongs to $\mathcal{T}_{1}$. Suppose that $\phi\left(x \otimes y_{0}\right)=u \otimes v$; it suffices to prove that $v$ is a multiple of a fixed vector $v_{0}$. We distinguish two cases.

Case 1: $\theta_{1}(E)<E$. Fix a unit vector $x_{0}$ in $E \ominus \theta_{1}(E)$. Then $x \otimes x_{0}$ and $x_{0} \otimes y_{0}$ belong to $\mathcal{T}_{1}$. Suppose that $\phi\left(x \otimes x_{0}\right)=f \otimes g$ and $\phi\left(x_{0} \otimes y_{0}\right)=u_{0} \otimes v_{0}$. Then we have that $u \otimes v=\phi\left(x \otimes y_{0}\right)=\phi\left(\left(x \otimes x_{0}\right)\left(x_{0} \otimes y_{0}\right)\right)=\left(u_{0}, g\right) f \otimes v_{0}$. Thus $v$ must be a multiple of $v_{0}$.

Case 2: $\theta_{1}(E)=E$. Then there is $F$ in $\mathcal{N}_{1}$ such that $(0)<F<E$.

By an argument similar to Case 1 , there is a fixed vector $v_{0}$ such that $\phi\left(x_{1} \otimes y_{0}\right)=$ $u_{1} \otimes v_{0}$ for every $x_{1} \in F$. For a non-zero vector $x_{2} \in E \ominus F$, suppose that $\phi\left(x_{2} \otimes y_{0}\right)=$ $u_{2} \otimes z$ and $\phi\left(x_{1} \otimes x_{2}\right)=f \otimes g$. Then $\left\|x_{2}\right\|^{2}\left(u_{1} \otimes v_{0}\right)=\phi\left(\left(x_{1} \otimes x_{2}\right)\left(x_{2} \otimes y_{0}\right)\right)=$ $\left(u_{2}, g\right) f \otimes z$. Thus $z$ is a multiple of $v_{0}$ and hence $\phi\left(x_{2} \otimes y_{0}\right)=u_{2}^{\prime} \otimes v_{0}$. Writing $x=x_{1}+x_{2} \in F \oplus(E \ominus F)$, we have that $\phi\left(x \otimes y_{0}\right)=\phi\left(\left(x_{1}+x_{2}\right) \otimes y_{0}\right)=$ $u_{1} \otimes v_{0}+u_{2}^{\prime} \otimes v_{0}=u \otimes v_{0}$.

Lemma 2.2. Suppose that $\bigvee\left\{\theta_{1}(E)^{\perp}: E \in \mathcal{N}_{1}^{\theta_{1}}\right\}=\mathcal{H}_{1}$ and $\phi$ preserves rank one operators. Let $E$ be in $\mathcal{N}_{1}^{\theta_{1}}$. If $x_{0}$ is a non-zero vector in $E$, then there is $u_{0}$ with the property that for every $y \in \theta_{1}(E)^{\perp}$ there exists a vector $v$ such that $\phi\left(x_{0} \otimes y\right)=u_{0} \otimes v$.

Proof. We distinguish two cases.

Case 1: $\theta_{1}(E)<E$. Let $y_{0}$ be a fixed unit vector in $E \ominus \theta_{1}(E)$. Then $x_{0} \otimes y_{0} \in \mathcal{T}_{1}$. Suppose that $\phi\left(x_{0} \otimes y_{0}\right)=u_{0} \otimes v_{0}$. For $y \in \theta_{1}(E)^{\perp}$, suppose that $\phi\left(x_{0} \otimes y\right)=u \otimes v$ and $\phi\left(y_{0} \otimes y\right)=f \otimes g$. Then we have that $u \otimes v=\phi\left(\left(x_{0} \otimes y_{0}\right)\left(y_{0} \otimes y\right)\right)=\left(f, v_{0}\right) u_{0} \otimes g$. It follows that $u$ must be a multiple of $u_{0}$.

Case 2: $\theta_{1}(E)=E$. Then there is $F$ such that $(0)<F<E$.

If $x_{0} \in F$, by an argument similar to Case 1 , the assertion holds.

Now assume that $z_{0}=(E-F) x_{0} \neq 0$. Fix $y_{0}$ in $\theta_{1}(E)^{\perp}$ and suppose that $\phi\left(x_{0} \otimes y_{0}\right)=u_{0} \otimes v_{0}$. For any $y_{1} \in \theta_{1}(E)^{\perp}$ which is linearly independent of $y_{0}$, suppose that $\phi\left(x_{0} \otimes y_{1}\right)=u_{1} \otimes v_{1}$. Since $u_{0} \otimes v_{0}+u_{1} \otimes v_{1}\left(=\phi\left(x_{0} \otimes\left(y_{0}+y_{1}\right)\right)\right)$ is a 
rank one operator, one of the pairs $\left\{u_{1}, u_{0}\right\}$ and $\left\{v_{1}, v_{0}\right\}$ must be linearly dependent. If $u_{1}$ and $u_{0}$ are linearly dependent, then we are done. If $u_{1}$ and $u_{0}$ are linearly independent, then $v_{1}$ and $v_{0}$ are linearly dependent. In this case, take $x_{1}$ in $F$. By an argument similar to Case 1 , we have that $\phi\left(x_{1} \otimes y_{i}\right)=f \otimes g_{i}(i=0,1)$. Suppose that $\phi\left(x_{1} \otimes z_{0}\right)=h \otimes w$. Then we have that $\left\|z_{0}\right\|^{2} f \otimes g_{i}=\phi\left(\left(x_{1} \otimes z_{0}\right)\left(x_{0} \otimes y_{i}\right)\right)=$ $\left(u_{i}, w\right) h \otimes v_{i}, i=0,1$, which implies that $g_{0}$ and $g_{1}$ are linearly dependent, and hence $y_{0}$ and $y_{1}$ are linearly dependent, which contradicts the hypothesis that $y_{0}$ and $y_{1}$ are linearly independent.

Let $\mathcal{N}$ be a nest over $\mathcal{H}$. Let $x$ be a non-zero vector in $\mathcal{H}$. Then $E_{x}=\bigwedge\{N \in$ $\mathcal{N}: x \in N\}$ is the smallest element in $\mathcal{N}$ to which $x$ belongs. We say that such an $E_{x}$ is the smallest element of $x$ in $\mathcal{N}$ and $x$ is a maximal vector of $E_{x}$. For a non-zero element $E$ in $\mathcal{N}$, we can construct a maximal vector $x_{E}$ of $E$ as follows. If $E \neq E_{-}$, then any non-zero vector $x_{E}$ in $E \ominus E_{-}$will be. If $E=E_{-}$, then there is an increasing sequence $\left\{E_{k}\right\} \subseteq \mathcal{N}$ such that $\lim _{k \rightarrow \infty} E_{k}=E$ in the strong operator topology. Let $e_{k}$ be a unit vector in $E_{k+1} \ominus E_{k}$. Then $x_{E}=\sum_{k=1}^{\infty} \frac{1}{2^{k}} e_{k}$ is a maximal vector of $E$.

Theorem 2.3. Suppose that $\bigvee\left\{\theta_{i}(E)^{\perp}: E \in \mathcal{N}_{i}^{\theta_{i}}\right\}=\mathcal{H}_{i}(i=1,2)$ and $\phi$ carries rank one operators to rank one operators. For each $E \in \mathcal{N}_{1}^{\theta_{1}}$, let $x_{E}$ be a fixed unit maximal vector of $E$. Then

(1) There is an order preserving map $E \rightarrow \widehat{E}$ from $\mathcal{N}_{1}^{\theta_{1}}$ to $\mathcal{N}_{2}^{\theta_{2}}$ such that $\bigvee\left\{\theta_{2}(\widehat{E})^{\perp}: E \in \mathcal{N}_{1}^{\theta_{1}}\right\}=\mathcal{H}_{2}$.

(2) For every $E \in \mathcal{N}_{1}^{\theta_{1}}$, there exists a unit vector $u_{E} \in \widehat{E}$ and a linear bijective map $A_{E}$ from $\theta_{1}(E)^{\perp}$ onto $\theta_{2}(\widehat{E})^{\perp}$ such that $\phi\left(x_{E} \otimes y\right)=u_{E} \otimes\left(A_{E} y\right)$ for any $y \in \theta_{1}(E)^{\perp}$.

(3) There is a linear bijective map $A$ from $\bigcup\left\{\theta_{1}(E)^{\perp}: E \in \mathcal{N}_{1}^{\theta_{1}}\right\}$ onto $\bigcup\left\{\theta_{2}(\widehat{E})^{\perp}: E \in \mathcal{N}_{1}^{\theta_{1}}\right\}$ such that $A T^{*}=\phi(T)^{*} A$ on $\bigcup\left\{\theta_{1}(E)^{\perp}: E \in \mathcal{N}_{1}^{\theta_{1}}\right\}$.

(4) Moreover, if both $\phi$ and $\phi^{-1}$ are bounded, then $A_{E}$ and $A$ are also bounded.

Proof. (1) Let $E$ be in $\mathcal{N}_{1}^{\theta_{1}}$. By Lemma 2.2, there is a fixed unit vector $u_{E}$ with the property that for every $y \in \theta_{1}(E)^{\perp}$ there is $v$ such that

$$
\phi\left(x_{E} \otimes y\right)=u_{E} \otimes v .
$$

Let $\widehat{E}$ be the smallest element of $u_{E}$ in $\mathcal{N}_{2}$. Then the map $E \rightarrow \widehat{E}$ is well defined.

Since $\mathcal{T}_{2}$ is a $\theta_{2}$-subalgebra, the vector $v$ in the right side of equation (2.1) is in $\theta_{2}(\widehat{E})^{\perp}$ for every $y \in \theta_{1}(E)^{\perp}$, which implies that $\widehat{E}$ is in $\mathcal{N}_{2}^{\theta_{2}}$.

Let $E_{1}$ and $E_{2}$ be in $N_{1}^{\theta_{1}}$ such that $E_{1}<E_{2}$. By the choice of $x_{E}$ 's, there is a vector $y_{1}$ in $E_{1}^{\perp}$ such that $\left(x_{E_{2}}, y_{1}\right) \neq 0$. Let $y_{2}$ be in $\theta_{1}\left(E_{2}\right)^{\perp}$. Then $x_{E_{1}} \otimes y_{1}$ and $x_{E_{2}} \otimes y_{2}$ belong to $\mathcal{T}_{1}$. Suppose that $\phi\left(x_{E_{i}} \otimes y_{i}\right)=u_{E_{i}} \otimes v_{i} \quad(i=1,2)$. Then $\left(u_{E_{2}}, v_{1}\right) u_{E_{1}} \otimes v_{2}=\left(x_{E_{2}}, y_{1}\right) \phi\left(x_{E_{1}} \otimes y_{2}\right) \neq 0$ and hence $\left(u_{E_{2}}, v_{1}\right) \neq 0$. Since $u_{E_{2}} \in \widehat{E_{2}}$ and $v_{1} \in \theta_{2}\left(\widehat{E_{1}}\right)^{\perp}$, we have that $\widehat{E_{2}} \theta_{2}\left(E_{1}\right)^{\perp} \neq 0$. Thus $\theta_{2}\left(\widehat{E_{1}}\right)<\widehat{E_{2}}$ and hence $\widehat{E_{1}} \leq \widehat{E_{2}}$.

Let $F$ be in $\mathcal{N}_{2}^{\theta_{2}}$. Let $u_{F}$ be a unit maximal vector of $F$. Applying Lemma 2.2 to $\phi^{-1}$, there is a unit vector $x_{F}$ with the property that for every $v \in \theta_{2}(F)^{\perp}$ there is $y(v)$ such that

$$
\phi\left(x_{F} \otimes y(v)\right)=u_{F} \otimes v .
$$


Let $E$ be the smallest element of $x_{F}$ in $\mathcal{N}_{1}$. Then the vector $y(v)$ in the left side of equation (2.2) is in $\theta_{1}(E)^{\perp}$. Hence $E \in \mathcal{N}_{1}^{\theta_{1}}$ and $x_{E} \otimes y(v) \in \mathcal{T}_{1}$. By equation (2.1), we have that

$$
\phi\left(x_{E} \otimes y(v)\right)=u_{E} \otimes v^{\prime}
$$

and $v^{\prime}$ is in $\theta_{2}(\widehat{E})^{\perp}$. By Lemma 2.1 and equations $(2.2)$ and $\left(2.1^{\prime}\right), v$ and $v^{\prime}$ must be linearly dependent, so $v \in \theta_{2}(\widehat{E})^{\perp}$. That is to say, for each $F \in \mathcal{N}_{2}^{\theta_{2}}$ there is $E \in \mathcal{N}_{1}^{\theta_{1}}$ such that $\theta_{2}(F)^{\perp} \subseteq \theta_{2}(\widehat{E})^{\perp}$. Thus

$$
\bigvee\left\{\theta_{2}(\widehat{E})^{\perp}: E \in \mathcal{N}_{1}^{\theta_{1}}\right\}=\bigvee\left\{\theta_{2}(F)^{\perp}: F \in \mathcal{N}_{2}^{\theta_{2}}\right\}=\mathcal{H}_{2}
$$

(2) By (1), for any $y \in \theta_{1}(E)^{\perp}$ there is a unique $v$ in $\theta_{2}(\widehat{E})^{\perp}$ such that $\phi\left(x_{E} \otimes y\right)=$ $u_{E} \otimes v$. Thus the map $A_{E}: y \rightarrow v$ is well defined, linear, from $\theta_{1}(E)^{\perp}$ into $\theta_{2}(\widehat{E})^{\perp}$. Moreover $\phi\left(x_{E} \otimes y\right)=u_{E} \otimes\left(A_{E} y\right)$ for any $y \in \theta_{1}(E)^{\perp}$. Now we prove that $A_{E}$ is onto.

Let $y_{0} \in \theta_{1}(E)^{\perp}$. Suppose that $\phi\left(x_{E} \otimes y_{0}\right)=u_{E} \otimes v_{0}$. For any $v$ in $\theta_{2}(\widehat{E})^{\perp}$, applying Lemma 2.2 to $\phi^{-1}$, there is a vector $y$ such that $x_{E} \otimes y \in \mathcal{T}_{1}$ and $\phi\left(x_{E} \otimes y\right)=$ $u_{E} \otimes v$. By the choice of $x_{E}$, we have that $y \in \theta_{1}(E)^{\perp}$.

(3) Fix $E_{0}$ in $\mathcal{N}_{1}^{\theta_{1}}$. Let $E$ be in $\mathcal{N}_{1}^{\theta_{1}}$. We want to prove that there is a scalar $\lambda_{E}$ such that

$$
\lambda_{E} A_{E} y=A_{E_{0}} y \text { on } \theta_{1}\left(E_{0}\right)^{\perp} \cap \theta_{1}(E)^{\perp} .
$$

If $\theta_{1}(E)<\theta_{1}\left(E_{0}\right)$, then both $x_{E} \otimes y$ and $x_{E_{0}} \otimes y$ belong to $\mathcal{T}_{1}$ for any $y \in \theta_{1}\left(E_{0}\right)^{\perp}$. Thus by (2) we have

$$
\begin{gathered}
\phi\left(x_{E} \otimes y\right)=u_{E} \otimes\left(A_{E} y\right), \\
\phi\left(x_{E_{0}} \otimes y\right)=u_{E_{0}} \otimes\left(A_{E_{0}} y\right) .
\end{gathered}
$$

Let $y_{0}$ be a fixed vector in $E_{0} \ominus \theta_{1}(E)$ such that $\left(y_{0}, x_{E_{0}}\right)=1$ (by the choice of $x_{E}$, such $y_{0}$ must exist). Then $u_{E} \otimes A_{E} y=\phi\left(\left(x_{E} \otimes y_{0}\right)\left(x_{E_{0}} \otimes y\right)\right)=\left(u_{E_{0}}, A_{E} y_{0}\right) u_{E} \otimes$ $A_{E_{0}} y$, and hence (2.3) holds. Likewise if $\theta_{1}(E)>\theta_{1}\left(E_{0}\right)$, there is also a scalar $\lambda_{E}$ such that equation (2.3) holds. If $\theta_{1}(E)=\theta_{1}\left(E_{0}\right)=F$, assume that $E<E_{0}$. For every $y \in F^{\perp}$, by $(2.5)$ and Lemma 2.1 ,

$$
\phi\left(x_{E} \otimes y\right)=u \otimes A_{E_{0}} y .
$$

Comparing (2.4) and (2.6), we deduce that $A_{E} y$ is a non-zero multiple of $A_{E_{0}} y$. Since this holds for every $y \in F^{\perp}$, it follows easily that $A_{E}$ is a non-zero multiple of $A_{E_{0}}$. That is, equation (2.3) holds.

Similarly, for any $E_{1}$ and $E_{2}$ in $\mathcal{N}_{1}^{\theta_{1}}$, there is a scalar $\lambda$ such that $\lambda A_{E_{1}} y=$ $A_{E_{2}} y$ on $\theta_{1}\left(E_{1}\right)^{\perp} \cap \theta_{1}\left(E_{2}\right)^{\perp}$. Hence there is a scalar $\mu$ such that $\mu \lambda_{E_{1}} A_{E_{1}} y=$ $\lambda_{E_{2}} A_{E_{2}} y$ on $\theta_{1}\left(E_{1}\right)^{\perp} \cap \theta_{1}\left(E_{2}\right)$. Since $\lambda_{E_{1}} A_{E_{1}} y=A_{E_{0}} y=\lambda_{E_{2}} A_{E_{2}} y$, on $\theta_{1}\left(E_{0}\right)^{\perp} \cap$ $\theta_{1}\left(E_{1}\right)^{\perp} \cap \theta_{1}\left(E_{2}\right)^{\perp}$, we have that $\mu=1$, that is,

$$
\lambda_{E_{1}} A_{E_{1}} y=\lambda_{E_{2}} A_{E_{2}} y \text { on } \theta_{1}\left(E_{1}\right)^{\perp} \cap \theta_{1}\left(E_{2}\right)^{\perp} .
$$

Define $A$ from $\bigcup\left\{\theta_{1}(E)^{\perp}: E \in \mathcal{N}_{1}^{\theta_{1}}\right\}$ to $\bigcup\left\{\theta_{2}(\widehat{E})^{\perp}: E \in \mathcal{N}_{1}^{\theta_{1}}\right\}$ by $A y=\lambda_{E} A_{E} y$ for $y \in \theta_{1}(E)^{\perp}$. By equation (2.7), $A$ is well defined and bijective. Moreover, we have $\phi\left(x_{E} \otimes y\right)=\bar{\lambda}_{E}^{-1} u_{E} \otimes(A y)$. 
Let $T \in \mathcal{T}_{1}$ and $y \in \theta_{1}(E)^{\perp}$, where $E \in \mathcal{N}_{1}^{\theta_{1}}$. We have $\bar{\lambda}_{E}^{-1} u_{E} \otimes\left(A T^{*} y\right)=$ $\phi\left(x_{E} \otimes T^{*} y\right)=\bar{\lambda}_{E}^{-1} u_{E} \otimes(A y) \phi(T)$. Hence $A T^{*} y=\phi(T)^{*} A y$. Since $y$ is arbitrary, we have that

$$
A T^{*}=\phi(T)^{*} A \text { on } \bigcup\left\{\theta_{1}(E)^{\perp}: E \in \mathcal{N}_{1}^{\theta_{1}}\right\} .
$$

(4) If $\phi$ and $\phi^{-1}$ are bounded, by (2) we have that $\left\|A_{E}\right\| \leq\|\phi\|$ and $\left\|A_{E}^{-1}\right\| \leq$ $\left\|\phi^{-1}\right\|$. By equation (2.3), $\left|\lambda_{E}\right| \leq\left\|A_{E}^{-1}\right\|\left\|A_{E_{0}}\right\| \leq\left\|\phi^{-1}\right\|\|\phi\|$. Consequently, by the definition of $A, A$ is bounded.

The next goal in this section is to give a sufficient condition such that an isomorphism of $\theta$-subalgebras preserves rank one operators and is bounded. To this end, we introduce the following concept.

Definition 2.4. Let $\mathcal{N}$ be a nest over a Hilbert space $\mathcal{H}$ and $\theta$ an order homomorphism from $\mathcal{N}$ into itself. The map $\theta$ is said to be dense if $\bigvee\{E \in \mathcal{N}: \theta(E)<$ $\mathcal{H}\}=\mathcal{H}$ and $\bigvee\left\{\theta(E)^{\perp}: E \in \mathcal{N}\right.$ and $\left.N>(0)\right\}=\mathcal{H}$.

Remark 2.5. (1) For a nest $\mathcal{N}$, it is well known that $\bigvee\left\{E \in \mathcal{N}: E_{-}<\mathcal{H}\right\}=\mathcal{H}$ and $\bigvee\left\{E_{-}^{\perp}: E \in \mathcal{N}\right.$ and $\left.E>(0)\right\}=\mathcal{H}$. Therefore, if $\theta(E)=E_{-}$for every $E \in \mathcal{N}$, then $\theta$ is dense.

(2) If $\mathcal{N}$ is quasi-continuous and $\theta$ is a map of $\mathcal{N}$ such that $E_{-} \leq \theta(E) \leq E$, then $(0) \leq \theta(E) \leq E<\mathcal{H}$ for every $(0)<E<\mathcal{H}$. Thus

$$
\bigvee\{E \in \mathcal{N}: \theta(E)<\mathcal{H}\} \geq \bigvee\{E \in \mathcal{N}: E<\mathcal{H}\}=\mathcal{H}
$$

and

$$
\bigwedge\{\theta(E): E \in \mathcal{N} \text { and } E>(0)\} \leq \bigwedge\{E \in \mathcal{N}: E>(0)\}=(0) .
$$

Hence $\theta$ is dense.

The following gives a characterization of rank one operators in a $\theta$-subalgebra, which assures that isomorphisms of $\theta$-subalgebras preserve rank one operators under the density assumption. Recall that an element $s$ of an abstract algebra $\mathcal{A}$ is called a single element of $\mathcal{A}$ if $a s b=0$ and $a, b \in \mathcal{A}$ implies that either $a s=0$ or $s b=0$ [6]. It is easy to see that every rank one operator is a single element of every operator algebra containing it.

Lemma 2.6. Let $\mathcal{T}$ be a $\theta$-subalgebra of $\mathcal{T}(\mathcal{N})$ and suppose $\theta$ is dense. Then every non-zero single element of $\mathcal{T}$ is of rank one.

Proof. For the case in which $\mathcal{T}$ is a large subalgebra (i.e. $\theta(E)=E_{-}$for every $E \in \mathcal{N}$ ), refer to [6. Lemma 2.3]. Here the proof is simpler. Suppose that $T$ has rank at least two. Since $\bigvee\{E \in \mathcal{N}: \theta(E)<\mathcal{H}\}=\mathcal{H}$, there is $E_{1}$ with $\theta\left(E_{1}\right)<\mathcal{H}$ such that $T E_{1}$ has rank at least two. Hence, since $\bigvee\left\{\theta(N)^{\perp}: N \in \mathcal{N}\right.$ and $\left.N>(0)\right\}=\mathcal{H}$, there is $E_{2}$ with $(0)<E_{2}$ such that $\theta\left(E_{2}\right)^{\perp} T E_{1}$ has rank at least two. Thus we can pick vectors $x_{1}$ and $x_{2}$ in $E_{1}$ such that $\theta\left(E_{2}\right)^{\perp} T x_{1}$ and $\theta\left(E_{2}\right)^{\perp} T x_{2}$ are non-zero and orthogonal. Take non-zero vectors $g$ in $\theta\left(E_{1}\right)^{\perp}$ and $h$ in $E_{2}$. Let $A=h \otimes\left(\theta\left(E_{2}\right)^{\perp} T x_{1}\right)$ and $B=x_{2} \otimes g$. Then both $A$ and $B$ belong to $\mathcal{T}$. It is easy to see that $A T x_{1} \neq 0, T B g \neq 0, A T B=\left(T x_{2}, \theta\left(E_{2}\right)^{\perp} T x_{1}\right) h \otimes g=0$. Namely, $T$ is not a single element of $\mathcal{T}$.

Here we give an example which shows that if $\theta$ is not dense, then Lemma 2.6 may not hold. Let $\mathcal{N}$ be a nest over $\mathcal{H}$ such that $E=(0)_{+}>(0)$. Then for each $S$ in $\mathcal{R}_{\mathcal{N}}$, the Jacobson radical of $\mathcal{T}(\mathcal{N})$, we have that $S E=E S E=0$. Let $T$ be 
in $\mathcal{R}_{\mathcal{N}}$ such that $T=E T E^{\perp}$. Then $S T=S E T=0$ for every $S \in \mathcal{R}_{\mathcal{N}}$. However such $T$ is not necessarily of rank one unless $E$ or $E^{\perp}$ is of dimension one.

Theorem 2.7. Suppose that $\phi$ is an isomorphism from a $\theta_{1}$-subalgebra $\mathcal{T}_{1}$ onto a $\theta_{2}$-subalgebra $\mathcal{T}_{2}$. If both $\theta_{1}$ and $\theta_{2}$ are dense, then $\phi$ carries rank one operators in $\mathcal{T}_{1}$ to rank one operators in $\mathcal{T}_{2}$.

Proof. By Lemma 2.6, it is a simple algebraic exercise. We omit it.

Lemma 2.8. Suppose that $\phi$ is an isomorphism from a $\theta_{1}$-subalgebra $\mathcal{T}_{1}$ onto a $\theta_{2}$-subalgebra $\mathcal{T}_{2}$ and $u_{i} \otimes v_{i}(i=1,2)$ belong to $\mathcal{T}_{2}$. If both $\theta_{1}$ and $\theta_{2}$ are dense, then $T \rightarrow\left(u_{1} \otimes v_{1}\right) \phi(T)\left(u_{2} \otimes v_{2}\right)$ is continuous on $\mathcal{T}_{1}$.

Proof. By Theorem 2.7, there are $x_{1} \otimes y_{1}$ and $x_{2} \otimes y_{2}$ in $\mathcal{T}_{1}$ such that $\phi\left(x_{i} \otimes y_{i}\right)=$ $u_{i} \otimes v_{i}$. Thus the continuity of $\left(u_{1} \otimes v_{1}\right) \phi(T)\left(u_{2} \otimes v_{2}\right)$ is immediate from

$$
\left(u_{1} \otimes v_{1}\right) \phi(T)\left(u_{2} \otimes v_{2}\right)=\phi\left(\left(x_{1} \otimes y_{1}\right) T\left(x_{2} \otimes y_{2}\right)\right)=\left(T x_{2}, y_{1}\right) \phi\left(x_{1} \otimes y_{2}\right) .
$$

Theorem 2.9. Suppose that $\phi$ is an isomorphism from a closed $\theta_{1}$-subalgebra $\mathcal{T}_{1}$ onto a closed $\theta_{2}$-subalgebra $\mathcal{T}_{2}$. If both $\theta_{1}$ and $\theta_{2}$ are dense, then $\phi$ is automatically continuous.

Proof. By the closed graph theorem, it suffices to prove that $\phi$ is a closed operator from $\mathcal{T}_{1}$ into $\mathcal{T}_{2}$. Let $T_{n}, T$ be in $\mathcal{T}_{1}$ and $S$ in $\mathcal{T}_{2}$ such that $T_{n} \rightarrow T$ and $\phi\left(T_{n}\right) \rightarrow S$.

Let $F$ be in $\mathcal{N}_{2}^{\theta_{2}}$ and $x$ in $F$. We want to prove that $\phi(T) x=S x$. In fact, take a non-zero vector $y$ in $\theta_{2}(F)^{\perp}$; then $x \otimes y$ is in $\mathcal{T}_{2}$. For any $F^{\prime} \in \mathcal{N}_{2}^{\theta_{2}}$, let $u$ be a fixed non-zero vector in $F^{\prime}$. Then for every $v \in \theta_{2}\left(F^{\prime}\right)^{\perp}, u \otimes v \in \mathcal{T}_{2}$. By the continuity of $(u \otimes v) \phi(\cdot)(x \otimes y)$ on $\mathcal{T}$, we obtain that $(u \otimes v) \phi(T)(x \otimes y)=(u \otimes v) S(x \otimes y)$, and hence $(\phi(T) x, v)=(S x, v)$. Since $\bigvee\left\{\theta_{2}(F)^{\perp}: F \in \mathcal{N}_{2}^{\theta_{2}}\right\}=\mathcal{H}_{2}$, we have that $\phi(T) x=S x$. Furthermore, since $\bigvee\left\{F: F \in \mathcal{N}_{2}^{\theta_{2}}\right\}=\mathcal{H}_{2}$, we have that $\phi(T)=S$.

\section{ISOMORPHISMS OF LARGE SUBALGEBRAS AND OF RADICAL-TYPE SUBALGEBRAS}

Theorem 3.1. Let $\mathcal{N}_{i}$ be a nest of subspaces of a Hilbert space $\mathcal{H}_{i}$ and $\mathcal{T}_{i}$ be a closed subalgebra of the nest algebra $\mathcal{T}_{i}\left(\mathcal{N}_{i}\right)$ associated to $\mathcal{N}_{i}(i=1,2)$. Let $\phi$ be an isomorphism from $\mathcal{T}_{1}$ onto $\mathcal{T}_{2}$. Then

(1) if $\mathcal{T}_{i}$ is large $(i=1,2)$, then $\phi$ is spatial;

(2) if $\mathcal{T}_{i}$ is radical-type and $\mathcal{N}_{i}$ is quasi-continuous $(i=1,2)$, then $\phi$ is spatial.

Proof. By Remark 2.5 and Theorem 2.7, $\phi$ carries rank one operators to rank one operators. Moreover by Theorem 2.9, $\phi$ is bounded and hence $\phi^{-1}$ is also bounded. Thus the bijective linear map $A$ provided by Theorem 2.3 is densely defined and has a dense range and is bounded. So it can be extended to be an invertible operator $V$ from $\mathcal{H}_{1}$ onto $\mathcal{H}_{2}$. By equation (2.8), we have that for $T \in \mathcal{T}_{1}, V T^{*}=\phi(T)^{*} V$ holds on $\mathcal{H}_{1}$. Therefore, $\phi(T)=V^{*-1} T V^{*}$.

The following corollaries are obvious.

Corollary 3.2. An isomorphism between the compact operator ideals of nest algebras is spatially implemented. 
Let $\mathcal{R}_{\mathcal{N}}$ and $\mathcal{K}_{\mathcal{N}}$ be the Jacobson radical and the compact operator ideal of the nest algebra $\mathcal{T}(\mathcal{N})$. Then $\mathcal{R}_{\mathcal{N}}+\mathcal{K}_{\mathcal{N}}$ is a closed subalgebra of $\mathcal{T}(\mathcal{N})$ and is called the compact perturbation of $\mathcal{R}_{\mathcal{N}}$ [4].

Corollary 3.3. An isomorphism between the compact perturbations of the Jacobson radicals of nest algebras is spatially implemented.

In the following, an (left or right) ideal $\mathcal{I}$ of a nest algebra $\mathcal{T}(\mathcal{N})$ is said to be diagonal-disjoint if $\mathcal{I} \cap\left(\mathcal{T}(\mathcal{N}) \cap \mathcal{T}(\mathcal{N})^{*}\right)=\{0\}$.

Lemma 3.4. Let $\mathcal{I}$ be a diagonal-disjoint (left or right) ideal of a nest algebra $\mathcal{T}(\mathcal{N})$. If $\mathcal{I}$ contains the Jacobson radical of $\mathcal{T}(\mathcal{N})$, then it is radical-type.

Proof. See Theorem 2.2 and Remark 3.8 in [5].

Corollary 3.5. Let $\mathcal{I}_{i}$ be a diagonal-disjoint closed (left or right) ideal of a nest algebra $\mathcal{T}\left(\mathcal{N}_{i}\right)$ such that it contains the Jacobson radical of $\mathcal{T}\left(\mathcal{N}_{i}\right)(i=1,2)$, and let $\phi$ be an isomorphism from $\mathcal{I}_{1}$ onto $\mathcal{I}_{2}$. If $\mathcal{N}_{i}$ is quasi-continuous, then $\phi$ is spatial.

It is well known that the Jacobson radical, the Larson ideal and $J_{\mathcal{N}}$ are diagonaldisjoint and contain the Jacobson radical. So the above corollary is rich. However, here is an example that shows that if a nest $\mathcal{N}$ is not quasi-continuous, then an automorphism of the Jacobson radical may not be spatial.

Example 3.6. Let $\mathcal{N}=\left\{(0)<E_{1}<E_{2}<E_{3}<\mathcal{H}\right\}$ be a nest over a Hilbert space $\mathcal{H}$. Then $T$ in the Jacobson radical $R_{\mathcal{N}}$ of $\mathcal{T}(\mathcal{N})$ is of the form

$$
T=\left[\begin{array}{cccc}
0 & T_{12} & T_{13} & T_{14} \\
0 & 0 & T_{23} & T_{24} \\
0 & 0 & 0 & T_{34} \\
0 & 0 & 0 & 0
\end{array}\right] \text { on } \mathcal{H}=E_{1} \oplus\left(E_{2} \ominus E_{1}\right) \oplus\left(E_{3} \ominus E_{2}\right) \oplus E_{3}^{\perp} .
$$

Let $L$ be an operator in $B\left(E_{1}\right)$ and $R$ be a non-zero operator from $E_{3}^{\perp}$ to $E_{2} \ominus E_{1}$. For $T \in R_{\mathcal{N}}$, define

$$
\phi(T)=\left[\begin{array}{cccc}
0 & T_{12} & T_{13} & T_{14}+L T_{12} R \\
0 & 0 & T_{23} & T_{24} \\
0 & 0 & 0 & T_{34} \\
0 & 0 & 0 & 0
\end{array}\right]
$$

It is easy to verify that $\phi$ is an algebraic automorphism of $R_{\mathcal{N}}$. But such $\phi$ is not spatial unless $L$ is a multiple of the identity on $E_{1}$. To see this, suppose that there is an invertible operator $S$ such that

$$
S T=\phi(T) S
$$

for every $T \in R_{\mathcal{N}}$. Suppose that $S=\left[S_{i j}\right]_{4 \times 4}$ on $\mathcal{H}=E_{1} \oplus\left(E_{2} \ominus E_{1}\right) \oplus\left(E_{3} \ominus\right.$ $\left.E_{2}\right) \oplus E_{3}^{\perp}$. Rewrite $S=\left[\begin{array}{cc}S_{1} & S_{2} \\ S_{3} & S_{4}\end{array}\right]$ on $\mathcal{H}=E_{2} \oplus E_{2}^{\perp}$. Substituting $T \in R_{\mathcal{N}}$ of the form $T=\left[\begin{array}{ll}0 & T_{1} \\ 0 & T_{2}\end{array}\right]$ on $\mathcal{H}=E_{2} \oplus E_{2}^{\perp}$ (i.e. $T_{12}=0$ ) to (3.1), noting that $\phi(T)=T$ for this case, we have $\left[\begin{array}{lll}0 & S_{1} T_{1}+S_{2} T_{2} \\ 0 & S_{3} T_{1}+S_{4} T_{2}\end{array}\right]=\left[\begin{array}{ccc}T_{1} S_{3} & T_{1} S_{4} \\ T_{2} S_{3} & T_{2} S_{4}\end{array}\right]$. Thus $T_{1} S_{3}=0$ for every $T_{1} \in B\left(E_{2}^{\perp}, E_{2}\right)$ and hence $S_{3}=0$. Let $T_{2}=0$. Then we have that $S_{1} T_{1}=T_{1} S_{4}$ for every $T_{1} \in B\left(E_{2}^{\perp}, E_{2}\right)$ and hence there is a scalar $\lambda$ such that $S_{1}=\lambda E_{2}$ and 
$S_{4}=\lambda E_{2}^{\perp}$. Hence $S_{2} T_{2}=0$, which forces that $S_{13}=0$ and $S_{23}=0$. Therefore

$$
S=\left[\begin{array}{cccc}
\lambda & 0 & 0 & S_{14} \\
0 & \lambda & 0 & S_{24} \\
0 & 0 & \lambda & 0 \\
0 & 0 & 0 & \lambda
\end{array}\right] .
$$

Substituting

$$
T=\left[\begin{array}{cccc}
0 & T_{12} & 0 & 0 \\
0 & 0 & 0 & 0 \\
0 & 0 & 0 & 0 \\
0 & 0 & 0 & 0
\end{array}\right]
$$

in (3.1) we obtain $T_{12} S_{24}+\lambda L T_{12} R=0$ for all $T_{12}$. Since $\lambda \neq 0, L$ must be a multiple of the identity on $E_{1}$.

Now we deal with isomorphisms of non-closed large subalgebras. As we have seen, when large subalgebras are closed, the boundedness of isomorphisms between them can be concluded by the Closed Graph Theorem. However, when large subalgebras are not closed, the Closed Graph Theorem is not applicable. In the following, we consider two special cases: nests are not quasi-continuous and large subalgebras contain masas.

Theorem 3.7. Let $\mathcal{T}_{1}$ and $\mathcal{T}_{2}$ be large subalgebras of nest algebras $\mathcal{T}\left(\mathcal{N}_{1}\right)$ and $\mathcal{T}\left(\mathcal{N}_{2}\right)$. Let $\phi$ be an isomorphism from $\mathcal{T}_{1}$ onto $\mathcal{T}_{2}$. If one of $\mathcal{N}_{1}$ and $\mathcal{N}_{2}$ is not quasi-continuous, then $\phi$ is spatial.

Proof. Without loss of generality, we assume that $\mathcal{N}_{1}$ is not quasi-continuous.

Case 1: $(0)_{+}>(0)$. Let $E \rightarrow \widehat{E}$ be the map defined in Theorem 2.3 from $\left\{E: E \in N_{1}\right.$ and $\left.E_{-}<\mathcal{H}_{1}\right\}$ to $\left\{F: F \in \mathcal{N}_{2}\right.$ and $\left.F_{-}<\mathcal{H}_{2}\right\}$. Then $\left(\widehat{(0)_{+}}\right)_{-}^{\perp}=$ $\bigvee\left\{(\widehat{E})_{-}^{\perp}: E \in \mathcal{N}_{1}\right.$ and $\left.E>(0)\right\}=\mathcal{H}_{2}$. By Theorem 2.3, there is a bijective linear map $A$ from $\mathcal{H}_{1}$ onto $\mathcal{H}_{2}$ such that

$$
\phi(T)^{*} A=A T^{*} .
$$

Now it suffices to prove that $A$ is bounded. Hence it suffices to prove that $A$ is a closed operator.

Let $\left\{x_{n}\right\} \subset \mathcal{H}_{1}$ such that $x_{n} \rightarrow x$ and $A x_{n} \rightarrow y$. We want to show that $A x=y$. For every rank one operator $S \in \mathcal{T}_{2}$, by (3.2) and the fact that $\phi$ preserves rank one operators, $S^{*} A$ is bounded. Thus $S^{*} A x_{n} \rightarrow S^{*} y$ and $S^{*} A x_{n} \rightarrow S^{*} A x$. Thus we have that $S^{*} A x=S^{*} y$ for every rank one operator $S \in \mathcal{T}\left(\mathcal{N}_{2}\right)$. Hence $S^{*} A x=S^{*} y$ for every finite rank operator $S \in \mathcal{T}\left(\mathcal{N}_{2}\right)$. By the density of finite rank operators in a nest algebra [2], there is a net $\left\{S_{\alpha}\right\}$ of finite rank operators in $\mathcal{T}\left(\mathcal{N}_{2}\right)$ such that $S_{\alpha}$ weakly converges to the identical operator on $\mathcal{H}_{2}$. For every vector $u$ in $\mathcal{H}_{2}$, it follows from $S^{*} x=S^{*} y$ that $\left(A x, S_{\alpha} u\right)=\left(y, S_{\alpha} u\right)$ and hence $(A x, u)=(y, u)$. Thus we have that $A x=y$.

Case 2: $\left(\mathcal{H}_{1}\right)_{-}<\mathcal{H}_{1}$. Let $\psi\left(T^{*}\right)=\phi(T)^{*}$ for every $T \in \mathcal{T}_{1}$. Then $\psi$ is an isomorphism from $\mathcal{T}_{1}^{*}$ onto $\mathcal{T}_{2}^{*}$, where $T_{i}^{*}=\left\{T^{*}: T \in \mathcal{T}_{i}\right\}(i=1,2)$. It is easy to see that $\mathcal{T}_{1}^{*}$ and $\mathcal{T}_{2}^{*}$ are large subalgebras of $\mathcal{T}\left(\mathcal{N}_{1}^{\perp}\right)$ and $\mathcal{T}\left(\mathcal{N}_{2}^{\perp}\right)$ respectively, where $\mathcal{N}_{i}^{\perp}=\left\{N^{\perp}: N \in \mathcal{N}_{i}\right\}$. Note that $(0)_{+}>(0)$ in $\mathcal{N}_{1}^{\perp}$ when $\left(\mathcal{H}_{1}\right)_{-}<\mathcal{H}_{1}$ in $\mathcal{N}_{1}$. By Case 1, there is a bounded and invertible operator $A$ such that $\psi\left(T^{*}\right)=A T^{*} A^{-1}$ and hence $\phi(T)=\left(A^{*}\right)^{-1} T A^{*}$ for every $T \in \mathcal{T}_{1}$. 
Theorem 3.8. Let $\mathcal{T}_{1}$ and $\mathcal{T}_{2}$ be large subalgebras of nest algebras $\mathcal{T}\left(\mathcal{N}_{1}\right)$ and $\mathcal{T}\left(\mathcal{N}_{2}\right)$. Let $\phi$ be an isomorphism from $\mathcal{T}_{1}$ onto $\mathcal{T}_{2}$. If $\mathcal{T}_{i}$ contains a masa, then $\phi$ is spatial.

Proof. By Theorem 3.7, we only need consider the case when both $\mathcal{N}_{1}$ and $\mathcal{N}_{2}$ are quasi-continuous. For this case, the result follows from Theorem 1.3.

There remains the following question on isomorphisms of large subalgebras:

Question 3.9. Let $\mathcal{N}$ be a quasi-continuous nest and $\mathcal{T}$ be the algebra of all finite rank operators in $\mathcal{T}(\mathcal{N})$. Let $\phi$ be an automorphism of $\mathcal{T}$. Is $\phi$ bounded?

\section{ACKNOWLEDGMENT}

The author would like to thank the referee for his very helpful comments and a careful reading of the paper.

\section{REFERENCES}

1. K. R. Davidson, Nest Algebras, Pitman Research Notes in Mathematics Series 191, Longman Scientific and Technical, Burnt Mill Harlow, Essex, UK, 1988. MR 90f:47062

2. J. A. Erdos, Operators of finite rank in nest algebras, J. London Math. Soc., 43(1968), 391-397. MR 37:5721

3. F. $\mathrm{Lu}$ and $\mathrm{S} . \mathrm{Lu}$, Isomorphisms between sub-strongly reducible maximal triangular algebras, Proc. Amer. Math. Soc., 128(2000), 2121-2128. MR 2001f:47117.

4. F. Lu and S. Lu, Perturbation of radical of nest algebras, Chinese Science Bulletin, 43(1998), 556-560. MR 99j:47063

5. F. Lu and S. Lu, Finite rank operators in some ideals of nest algebras, Acta Math. Sinica, 41(1998), 89-95. MR 2000c: 47129

6. J. R. Ringrose, On some algebras of operators II, Proc. London Math. Soc., 16(1966), 385-402. MR 33:4703

Department of Mathematics, Suzhou University, Suzhou 215006, People's Republic OF CHINA

E-mail address: fylu@pub.sz.jsinfo.net 